\title{
SNS ACCELERATOR DIAGNOSTICS: PROGRESS AND CHALLENGES*
}

\author{
T. J. Shea, S. Assadi, R. Witkover, Oak Ridge National Laboratory \\ L. Doolittle, D. Oshatz, Lawrence Berkeley National Laboratory \\ M. Plum, M. Stettler, J. O'Hara, J. Power, R. Shafer, Los Alamos National Laboratory \\ P. Cameron, R. Connolly, D. Gassner, M. Kesselman, Brookhaven National Laboratory
}

\section{Abstract}

A collaboration of six national laboratories, the Spallation Neutron Source (SNS) Project is constructing an accelerator based neutron facility sited in Oak Ridge, Tennessee. The goal of $2 \mathrm{MW}$ beam power, 95\% availability, and hands-on maintenance will place significant demands on the performance and operational reliability of accelerator diagnostics. Mirroring the project organization, a cross-laboratory team is developing diagnostic systems for the facility. This talk reports the team's progress and summarizes its approach to outstanding technical and organizational challenges.

\section{THE SNS PARTNERSHIP}

In Figure 1, the layout of the SNS facility is depicted on the Oak Ridge National Laboratory (ORNL) site. The major parameters are listed in Table 1 .

Lawrence Berkeley National Lab is responsible for the design and construction of the Front End System[1]. This system includes the $\mathrm{H}$ - ion source, an electrostatic low energy beam transport line (LEBT), a four-module radio frequency quadrupole (RFQ), and a medium energy beam transport line (MEBT). Besides matching the $65 \mathrm{keV}$ ion beam transversely into the RFQ, the LEBT also provides a beam chopping function with about 50 ns rise and fall times. The MEBT matches the bunched, $2.5 \mathrm{MeV}$ beam transversely and longitudinally into the Drift Tube Linac (DTL). Space is also provided for a chopper with a few ns rise time and for several diagnostic systems.

Los Alamos National Laboratory is providing a linac that consists of a $402.5 \mathrm{MHz}$ DTL, an $805 \mathrm{MHz}$ Coupled Cavity Linac (CCL), and an $805 \mathrm{MHz}$ Superconducting Linac (SCL)[2]. For the SCL, Thomas Jefferson National Accelerator Facility (Jlab) will provide the niobium cavities and their cryogenic systems.

From the linac, the H- beam enters either a low power tuning dump or the high-energy beam transport line (HEBT). Besides transporting the beam to the ring injection foil, HEBT also provides longitudinal manipulation, collimation, and measurement functions. Through charge changing injection into the ring, beam is accumulated into one long proton bunch. The ring RF systems keep a longitudinal gap clear so that an extraction kicker can send the bunch down the Ring to Beam Target

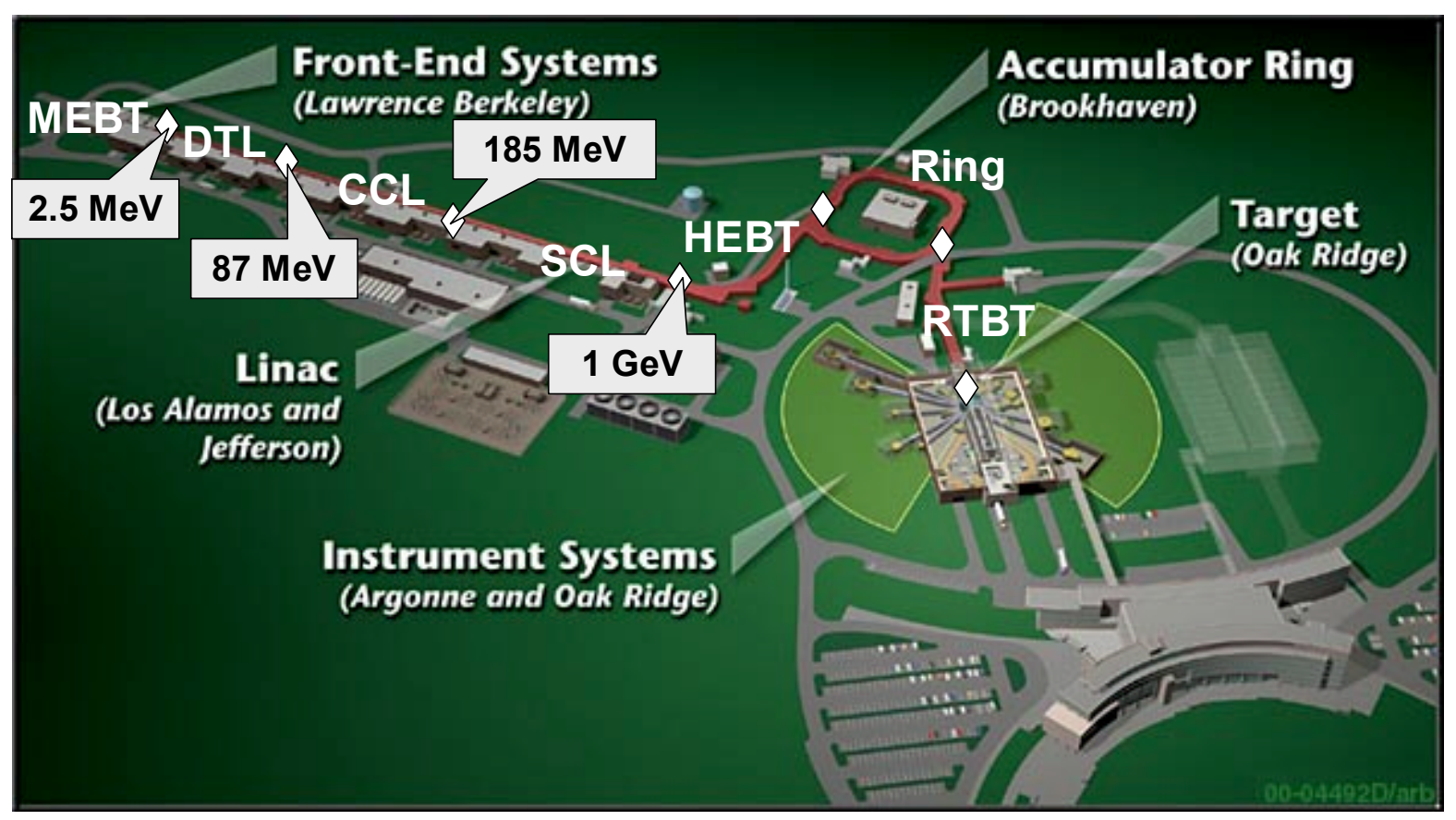

Figure 1: Layout of the SNS facility

*Work supported by U.S. Department of Energy. 
line (RTBT) to either a low power dump or to the liquid mercury target. Brookhaven National Lab is providing the HEBT, Ring, and RTBT.

ORNL is constructing the high power mercury target system while the neutron instruments are developed collaboratively by ORNL and Argonne. ORNL is responsible for overall project coordination and will eventually lead installation, commissioning, and operations.

\section{OVERVIEW OF ACCELERATOR DIAGNOSTICS}

For each accelerator section, Figure 2 lists the suite of diagnostic systems and their channel counts $[3,4]$. As described in Section 4, additional systems will be provided for early commissioning of the Front End and the DTL.

All systems are being designed and constructed by the multi-lab diagnostics team. The team is comprised of groups from BNL, LANL, LBNL, and ORNL. The ORNL group coordinates the team's activities. A Diagnostics Advisory Committee provides external guidance. This committee reviews all major system designs at the conceptual and detailed design stages.

\section{CHALLENGES AND STRATEGIES}

\section{Multi-lab organization}

The diagnostics team consists of four groups that are based in laboratories with strong traditions. In addition,
Table 1: Parameter List

\begin{tabular}{|l|l|}
\hline Parameter & Value \\
\hline Power on target & $1.4 \mathrm{MW}$ \\
\hline Proton beam energy on target & $1.0 \mathrm{GeV}$ \\
\hline Proton beam current on target & $1.4 \mathrm{~mA}$ \\
\hline Pulse repetition rate & $60 \mathrm{~Hz}$ \\
\hline Beam macropulse duty factor & $6 \%$ \\
\hline Avg. current in macro-pulse & $26 \mathrm{~mA}$ \\
\hline H- peak current, front end & $38 \mathrm{~mA}$ \\
\hline Chopper beam-on duty factor & $68 \%$ \\
\hline RFQ output energy & $2.5 \mathrm{MeV}$ \\
\hline Front end + linac length & $335 \mathrm{~m}$ \\
\hline DTL output energy & $87 \mathrm{MeV}$ \\
\hline CCL output energy & $185 \mathrm{MeV}$ \\
\hline SC linac output energy & $1 \mathrm{GeV}$ \\
\hline HEBT length & $170 \mathrm{~m}$ \\
\hline Accumulator ring circumference & $248 \mathrm{~m}$ \\
\hline Ring fill time & $1 \mathrm{~ms}$ \\
\hline Ring beam extraction gap & $250 \mathrm{~ns}$ \\
\hline RTBT length & $150 \mathrm{~m}$ \\
\hline Protons per pulse on target & $1.5 \mathrm{x} 10^{14}$ \\
\hline Pulse length on target & $695 \mathrm{~ns}$ \\
\hline Target material & $\mathrm{Hg}$ \\
\hline
\end{tabular}

individual group members have typically worked on intralaboratory projects that benefit from rapid, local coordination. Fortunately, there are examples of successful and extensive collaborations in high-energy physics and more recently, open source software projects.

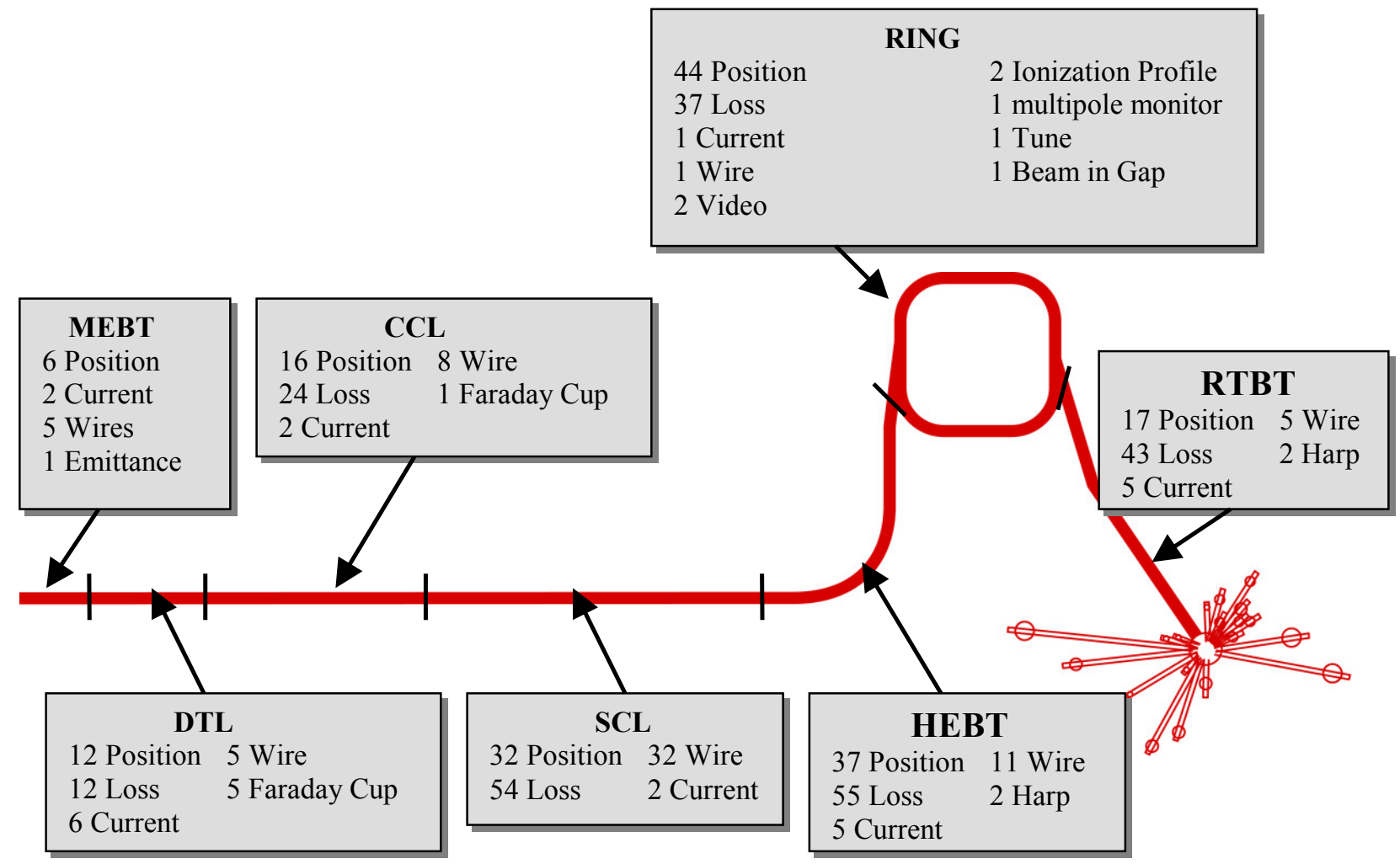

Figure 2. Layout of diagnostic systems 
Although we are still on the learning curve, several key strategies have already emerged. The diagnostics organization has evolved into a team of optimally coupled groups. The ORNL group attempts to provide adequate leadership and coordination without over constraining the groups from the partner laboratories (BNL, LANL, and LBNL). Although these partner labs will eventually deliver all systems to Oak Ridge, a strict customerprovider relationship is avoided. For each system, leadership is shared between ORNL and the partner labs. This encourages shared ownership of system requirements as well as the resulting designs. Currently, the system leads are developing handoff strategies that clarify responsibilities and define success.

Obviously, communication is a critical issue. Our primary tools are typical: phone conferencing, video conferencing, frequent email, and weekly written reports. When resolving difficult issues, nothing can replace faceto-face communication. Therefore, team members must travel frequently and continuously renew the trust of their colleagues.

The multi-laboratory environment also has inherent advantages. Some peer review occurs naturally in collaboration. Also, each group contributes particular technical strengths that may be lacking in another. By working with their colleagues from other labs, team members are continuously exposed to new ideas that are applicable at SNS and their home laboratories. Over the past two years, many common components and technologies have been identified and work on each one has been consolidated at one lab. This should result in efficiency and higher quality.

\section{Beam Characteristics}

A key feature of the SNS accelerator facility is the high beam power delivered to the target. Table 1 captures other key beam parameters. The head of the pulse is softened via pulse width modulation in order to reduce beamloading transients. Also, it is expected that some commissioning activities will employ single, $600 \mathrm{~ns}$ minipulses. Therefore, many of the diagnostic systems must possess a bandwidth of at least a few MHz. This will allow measurement of short bursts, and measurement of beam parameters during the transient. Also during commissioning, the peak current observed by a diagnostic may be reduced by an order of magnitude due to unintentional beam loss, or intentional current reduction. This leads to an additional dynamic range requirement on the linac diagnostics.

In the ring, the accumulation cycle leads to a far more challenging dynamic range requirement. During the onemillisecond pulse, the beam current will change by $60 \mathrm{~dB}$. Ring systems must accommodate this change while providing their specified signal to noise ratio. In most cases, the electronics will switch gains during the pulse.

Intense beams present problems for interceptive diagnostics and the SNS is no exception. Of particular concern is the wire scanners located in the linac. When these devices are in use, the run permit system will lock the machine into a short pulse mode. This system, in concert with the machine protect system[5,6], will protect all devices that might intercept the beam. Several diagnostic systems provide inputs to the MPS, including loss monitors, beam current monitors, and harps. Since failure of these diagnostics could lead to unplanned downtime, they must be designed for reliability.

In the ring, high beam intensity impacts the diagnostics in additional ways. An e-p instability could limit performance, so the beam pipe and many diagnostics devices will be TiN coated in an effort to suppress secondary emission. Space charge will significantly affect the tune footprint, so we are investigating ways to measure the incoherent tune. To reduce the space charge forces, transverse painting is employed. This will cause a significant increase in beam size during accumulation, making profile measurement challenging.

\section{Network Attached Devices}

Over the past few decades, some general trends in instrumentation electronics can be identified. The 1970s saw many modular systems that included simple register based cards services by centralized computers. By the 1980s, microprocessors had migrated into the crates and even onto intelligent acquisition cards, allowing some limited data processing before results were presented to the network. In the 1990s, many instrumentation systems could take advantage of higher speed digitizers that sampled above Nyquist. Dedicated signal processors were deployed to acquire the streaming data and provide functions that were formerly implemented in analog circuitry. Devices still shared a crate and its many resources. Modularity was implemented by function.

Most SNS diagnostic channels will be deployed as network attached devices (NADs). This is an evolution of architectures described above. In its ultimate form, a selfcontained NAD serves each pickup or sensor and interfaces to Ethernet, event links, and machine protect. These well-defined interfaces provide a loosely coupled architecture that reduces system brittleness. The NAD provides nearly all functions required by a particular device and can be serviced without affecting other devices. Many SNS diagnostics systems will utilize commercial off the shelf software as well as hardware. Current prototypes are based on PCI cards installed in rack mount PC servers. The architecture will evolve as PCs incorporate new technologies.

\section{PROGRESS}

\section{Beam Position Monitors}

Two styles of beam position monitors (BPMs) will be deployed: Linac and Ring. The Linac style $[7,8]$ will be used in the MEBT, DTL, CCL, SCL, and HEBT, where strong signals are expected at the $402.5 \mathrm{MHz}$ bunching frequency its first harmonic at $805 \mathrm{MHz}$. In these areas, the pickups are four electrode, 50-ohm, shorted microstrips. The linac style electronics uses an in-phase 
and quadrature phase (I-Q) receiver to make a full vector measurement of the four signals from the electrodes. As a result, this system can be used for beam phase measurements as well as for position measurements.

The ring style system is deployed where the linac bunches will have coalesced into a long bunch captured by the ring RF system. This beam is present in the ring and RTBT. In these areas, the pickups are four electrode, 50 -ohm, open microstrips. The signal is acquired at baseband by a wideband digitizing system with programmable gain.

Prototypes of nearly all pickups have been constructed. The electronics will be deployed as a NAD, using rack mount PC servers. Prototype linac systems have been tested and six units will be used at Berkeley during front end commissioning in 2002. Further design refinements will be based on this experience. The ring systems will be identical except for the analog front end to be prototyped in 2002 .

\section{Beam Loss Monitors}

The SNS will have two types of beam loss monitor: slow and fast[12,13,14,15,16]. The slow monitor system currently specifies an ion chamber similar to the RHIC design. This chamber provides a prompt signal due to the electrons. Unfortunately, a long tail due to the slow ion collection time follows this signal. A faster parallel plate design is being explored. A prototype analog front end has been constructed and a conceptual architecture based on commercial VME hardware has been developed. Design work on the fast system will follow in late 2001 and 2002.

\section{Video Foil Monitor}

Beam is injected into the SNS ring through a thin stripping foil. About $95 \%$ of the beam is stripped from $\mathrm{H}$ to protons and is successfully injected. The remaining fraction is stripped to $\mathrm{H} 0$ or misses the foil entirely. This residual beam is stripped by a second foil and is then transported to the ring injection dump. Light emitted from the beam spot on these foils is monitored by two video systems. Although the light intensity in the visible spectrum may not accurately represent the beam intensity, a simple visual view of the injection point has proven useful in the past.

Work on this system will begin in about one year. Since the injection area will see a high radiation dose, shielding of the cameras is a concern. We expect to use the off the shelf image processing hardware and software available on the PC platform. The spectral content of the light will be analyzed with the goal of achieving a more accurate current density measurement.

\section{Beam Current Monitors}

The pick-up element is a Bergoz ${ }^{\circledR}$ FCT (fast current transformer[9,10,11]. This transformer has been chosen with 50 turns, providing a trade-off between sensitivity, rise-time and droop. A droop of $0.1 \% / \mu$ s, rise time of $<1 \mathrm{~ns}$, and sensitivity of $0.5 \mathrm{~V} / \mathrm{A}$ is the target goal. The device has been built with an additional 10 turn winding to provide a calibration/test input capability. A first article has been received and tested.

The BCM electronics will be based on the same data acquisition platform as the position monitors. A programmable gain front end has been prototyped and integrated into this PC based platform. Two units are being prepared for delivery to LBNL in late 2001. They will be used during front end commissioning. The experience gained will be used to refine the design of the production hardware and software.

\section{Wire Scanners}

Two types of wire scanners are under development. The baseline device is a traditional stepping wire that intercepts the beam[17,18]. The other is a more experimental device that scans a laser across the ion beam and detects the effect of neutralization. The second device can only be used in the MEBT, linac, and HEBT.

In the traditional wire scanner, an actuator assembly is used to scan a series of wires through the beam. Both $X$ and Y-axis may be scanned with the same actuator, as well as a third axis at 45 degrees. The amount of beam current intercepted by the wire(s) is read out directly, or by measuring the beam loss on a nearby beam loss monitor (BLM). The measured wire signal may be normalizes to the beam current as measured with a nearby current monitor.

The laser profile monitor is being developed at Brookhaven[19] National Lab. Electrons can be removed from an $\mathrm{H}^{-}$beam by passing light from a near-infrared laser through it. Experiments have been performed on the BNL linac to measure the transverse profile of a $750 \mathrm{keV}$ beam by using a Nd:YAG laser to photoneutralize narrow slices of the beam. The laser beam is scanned across the ion beam neutralizing the portion of the beam struck by the laser. The electrons are removed from the ion beam and the beam current notch is measured. Additional tests are planned during 2001 in the BNL $200 \mathrm{MeV}$ line and during front end commissioning in 2002 at LBNL.

\section{Harps}

Harps, also known as multiwires, are located before the linac dump, injection dump, extraction dump, and target. Besides serving as a tuning aid, they also provide a machine protect function to avoid off normal beam pulses of excessive current density. The SNS harps will based on a proven LANSCE design in use at LANL. Each will have three measurement planes. Current design efforts are centered on wire lifetime and MPS integration.

\section{Faraday Cups}

Faraday cups will be mounted on actuators throughout the DTL. They will each include thin energy degrading foil to filter out low energy particles. A conceptual design 
has been developed. The bandwidth of these cups is not enough to measure bunch length, so a broadband faraday cup is under development. INFN-LNL in Legnaro, Italy is collaborating on this $30 \mathrm{GHz}$ device.

\section{Miscellaneous Ring Systems}

Design work on some systems will begin in late 2001 and 2002.

Beam in Gap: A kicker system will be installed in the ring to clear the extraction gap. Protons will be kicked transversely and intercepted by the betatron collimator.

Electron monitors: Electron production will be measured at several locations in the ring. The monitors must be insensitive to the fields produced by the circulating beam.

\section{Tune Measurement}

Several techniques will be used to measure tune in the ring. Coherent tune will first be measured by acquiring turn-by-turn data with the ring BPMs. This technique will rely on injection oscillations of a single minipulse. A kicker and dedicated pickups are also planned and will be used to measure beam transfer functions. Although incoherent tune is an important parameter, so far no reliable technique has been selected to measure it. This remains an $\mathrm{R} \& \mathrm{D}$ topic.

\section{Ionization Profile Monitor}

An ionization profile monitor similar to the one developed for RHIC will be deployed in the ring. One serious problem must be overcome. The space charge fields of the circulating beam leads to a large energy spread of the collected electrons. Because the microchannel plate has an energy dependent efficiency, this energy spread can lead to distortions of the measured profile. R\&D is underway to quantify this effect and to possibly develop alternative detection methods.

\section{Temporary Diagnostics}

A diagnostics station called D-plate, is being built to commission the SNS linac up through the first DTL tank[20]. Conceptual review of this system is complete and detailed design is in progress. A similar diagnostics beamline is currently under construction support of front end commissioning. The emittance scanner from this beamline will first be used in December 2001 to characterize beam from the RFQ.

\section{REFERENCES}

[1] Roderich Keller, "Progress with the SNS Front-End Systems", these proceedings.
[2] J. Stovall, J. Billen, K. Crandall, S. Nath, H. Takeda, R. Shafer, L. Young," Expected Beam Performance of the SNS Linac", these proceedings.

[3] T.J. Shea, et.al., "SNS Diagnostics", P. 132, Beam Instrumentation 9th Workshop-2000, Cambridge, AIP Conference Proceedings 546, MA, USA

[4] R. Hardekopf, S. S. Kurennoy, J.F. Power, R.E. Shafer, and J.E. Stovall, "Beam Diagnostic Suite for the SNS LINAC", P. 410, Beam Instrumentation 9th Workshop-2000, Cambridge, AIP Conference Proceedings 546, MA, USA.

[5] C.Sibley, "SNS Machine Protection System VME/PMC Module Design", Internal documentation: (http://www.sns.gov/projectinfo/ics/192/1923/MPS_F $\mathrm{DR} /$ )

[6] C.Sibley, "SNS System Requirements Document for Machine Protection System", Internal documentation: (http://www.sns.gov/projectinfo/ics/192/1923/ mpsInterfaceRequirements.doc)

[7] J. Power et. al., "Beam Position Monitors for the SNS Linac", these proceedings.

[8] S. Kurennoy," Effects of Transverse Beam Size in Beam Position Monitors", these proceedings.

[9] M. Kesselman, "Spallation Neutron Source Beam Current Monitor Electronics", these proceedings.

[10] M. Kesselman, R. Witkover, L. Doolittle, J. Power, "SNS Project-Wide Beam Current Monitors", ", P. 464, Beam Instrumentation 9th Workshop-2000, Cambridge, AIP Conference Proceedings 546, MA, USA.

[11]L. Young," A Procedure to Set Phase and Amplitude of the RF in the SNS Superconducting Cavities", these proceedings.

[12]David Gassner, "Spallation Neutron Source Beam Loss Monitor System", these proceedings.

[13]D. Gassner, R. Witkover, P. Cameron, J. Power, "Spallation Neutron Source Beam Loss Monitor System", P. 392, Beam Instrumentation 9th Workshop-2000, Cambridge, AIP Conference Proceedings 546, MA, USA.

[14] R. L. Witkover, R. J. Michnoff, J.M. Geller," RHIC Beam Loss Monitor System Initial Operation", P. 2247, Proceeding of the PAC 1999.

[15] R.A. Hardekopf, Beam Loss and Activation at LANSCE and SNS" LA-UR-RR-6825, Los Alamos National Laboratory, Los Alamos, NM, Sept. 1999.

[16]R.A. Shafer, "Beam Loss From H-minus Stripping in the residual Gas", SNS Technical Note LA-UR-992497, May 1999.

[17] Peter Cameron, "Carbon Wire Profile Monitors in the SNS", these proceedings.

[18] R. Hardekopf, R. Meyer, Sr., M. Plum, J. Power, D. Sattler, R. Shafer ," Wire-Scanner Design for the SNS Super conducting RF Linac", these proceedings

[19] R. Connolly, et. al., "Laser Profile Measurements of an H- Beam", these proceedings.

[20]M. Plum, R. Shafer, "Diagnostics Plate for SNS Linac Commissioning", these proceedings. 